\title{
GEOSPATIAL ASSESSMENT AND MODELING OF URBAN HEAT ISLANDS IN QUEZON CITY, PHILIPPINES USING OLS AND GEOGRAPHICALLY WEIGHTED REGRESSION
}

\author{
C. A. Alcantara ${ }^{1}$, J. D. Escoto ${ }^{1}$, A.C. Blanco ${ }^{1,2}$, A.B. Baloloy ${ }^{2}$, J.A. Santos ${ }^{2}$, R.R. Sta. Ana ${ }^{2}$ \\ ${ }^{1}$ Dept. of Geodetic Engineering, University of the Philippines, Diliman, Quezon City 1101, Philippines \\ caalcantara@up.edu.ph, jimescoto@outlook.com \\ ${ }^{2}$ Training Center for Applied Geodesy and Photogrammetry, University of the Philippines, Diliman, Quezon City 1101 \\ acblanco@up.edu.ph
}

KEY WORDS: UHI, UTFVI, Land Surface Temperature, NDBI, NDVI

\begin{abstract}
:
Urbanization has played an important part in the development of the society, yet it is accompanied by environmental concerns including the increase of local temperature compared to its immediate surroundings. The latter is known as Urban Heat Islands (UHI). This research aims to model UHI in Quezon City based on Land Surface Temperature (LST) estimated from Landsat 8 data. Geospatial processing and analyses were performed using Google Earth Engine, ArcGIS, GeoDa, and SAGA GIS. Based on Urban Thermal Field Variance Index (UTFVI) and the normalized mean per barangay (village), areas with strong UHI intensities were mapped and characterized. high intensity UHIs are observed mostly in areas with high Normalized Difference Built-up Index (NDBI) like the residential regions while the weak intensity UHIs are noticed in areas with high Normalized Difference Vegetation Index (NDVI) near the La Mesa Reservoir. In the OLS regression model, around 69\% of LST variability is explained by Surface Albedo (SA), Sky View Factor (SVF), Surface Area to Volume Ratio (SVR), Solar Radiation (SR), NDBI and NDVI. OLS yield relatively high residuals (RMSE $=1.67$ ) and the residuals are not normally distributed. Since LST is non-stationary, Geographically Weighted Regression (GWR) regression was conducted, proving normally and randomly distributed residuals (average RMSE = $0.26)$.
\end{abstract}

\section{INTRODUCTION}

There has been a drastic increase in the world population in recent years; new megacities are born, and existing megacities become more populated (Mirzaei, 2015). The increase in population tends to affect the environmental situations not only globally but also locally. According to the United Nations, 55\% of the current population around the world lives in urban areas. Urban land is predicted to cover $31 \%$ of the total land surface by 2027 according to Myronidis and Iannou (2018).

One of the key environmental climate variables defined and accepted by the Global Climate Observing System (GCOS) is land surface temperature (LST), which is used to determine Surface UHI. LST can be derived from thermal infrared (TIR) data that is used in surface energy balance models involving drought, evapotranspiration, hydrological cycles and climate research. It is also used in studying heat-related issues, impact of heat stress on the urban population and outbreak and propagation of vector-borne diseases (Malakar, 2018).

Urban heat island (UHI) is a worldwide phenomenon commonly observed describing urban areas with relatively higher temperatures than its surroundings (Lettenmaier, Mishra, Ganguly \& Nijssen, 2014). UHI is known to affect local weather and climate and impacts urban development and human living environments (Zhang \& Liu, 2011). Aside from the effect on temperature, UHIs can produce secondary effects on local meteorology, including the altering of local wind patterns, the development of clouds and fog, the humidity, and the rates of precipitation. (Trishita, 2017)
UHI has the potential to directly influence the health and welfare of urban residents. Increased temperatures have been reported to cause heat stroke, heat exhaustion, and heat cramps. Individuals with cognitive health issues are more at risk when faced with high temperatures. Also, people with other health problems like diabetes, obesity, have sleep deprivation, or have cardiovascular/cerebrovascular conditions should avoid too much heat exposure (Schneider \& Breitner, n.d.).

Another consequence of UHI is the increased energy required for air conditioning and refrigeration in cities that are in comparatively hot climates. The results of this research will provide the local government units some useful information about the critical factors that causes the formation of urban heat islands and its effects to human health. These factors shall be monitored and shall be properly considered in urban planning and development and reduce the effect of urban heat islands. (Lodi et al., 2014)

\subsection{Research objectives}

This research aims to assess the development of urban heat islands in rapidly urbanizing and highly urbanized cities in the Philippines and develop models for estimating land surface temperatures (LST) and predicting UHIs by relating LST with environmental factors, including land use land cover distribution, using regression modelling.

\subsection{Significance of the study}

The study on the assessment and modelling of urban heat island will help us determine the areas that are significantly affected by 
the phenomenon through the use of geographically available data. The methodologies presented in this research shall be helpful in determining or measuring land surface temperature and other factors using automated tools such as the Google Earth Engine without downloading big data.

\subsection{Study area}

Quezon City is a highly urbanized area located in the mid to northern part of the National Capital Region (NCR); it extends from 14.589 to 14.76 latitude and 120.986 to 121.131 longitude. The city covers the largest land area of $161.12 \mathrm{sq} . \mathrm{km}$ within the region or $25 \%$ or the total area of the region. Also has the largest total population of over 2.9 million according as of 2015 Census of Population. It is composed of 142 barangays separated within 6 legislative districts. The city is mostly composed of residential areas and commercial to industrial areas that makes the city to be highly urbanized and a suitable area of our study. As per World Population Review 2019, the city has an average population density of approximately 18,000 residents per square $\mathrm{km}$. Also, it has a recent annual population growth rate of more than $2 \%$ making the city's population to grow over the years with approximately over 3 million in 2020 .

\section{REVIEW OF RELATED LITERATURE}

\subsection{Causes of UHI}

One of the crucial topics in urban climatology studies is the UHI phenomenon. It is a complex feature which is described by the combination of green vegetation, water surfaces, impervious surface materials and exposed soils, resulting in a temporally and spatially varying LST. The characteristics of the city are probable causes of the heat island effect (Zhou \& Rbyski, 2017). These characteristics can be described by built-up index, vegetation index, surface albedo, sky view factor, and surface area to volume ratio.

\subsubsection{Sky View Factor (SVF)}

The sky view factor (SVF) is a dimensionless value which represents the ratio at a point in space between the visible sky and a hemisphere (Oke, 1981) which ranges between 0 and 1, representing totally obstructed and open spaces, respectively (Oke, 1988). SVF was found to be a strong causation factor for UHI which affects the temperature by analyzing the heat island intensities in the four typical areas in Montreal (Wang \& Akbari, 2014). Another study by Hammerle et al. (2009) concludes that the urban surface geometry described as SVF is a significant factor of the air temperature distribution inside the city.

\subsubsection{Surface Area-to-Volume Ratio (SVR)}

One important factor in determining heat loss and gain is the surface area-to-volume ratio (SVR). Heat loss/gain through a surface is directly proportional to its area. Small SVR imply minimum heat gain and minimum heat loss (CLEAR, n.d.).

\subsubsection{Surface Albedo (SA)}

Surface albedo (SA) is the fraction of incoming solar radiation at a surface (i.e. land, cloud top) that is effectively reflected by that surface. (American Meteorological Society, n.d.)

\subsubsection{Solar Radiation (SR)}

Solar radiation (SR) or solar resource is a term which refers to the electromagnetic radiation emitted by the sun. The magnitude of surface urban heat islands varies with seasons, due to changes in the sun's intensity as well as ground cover and weather. As a result of such variation, surface UHI are typically largest in the summer (Reducing Urban Heat Islands, n.d.). This means that temperature is also dependent on SR.

\subsubsection{Normalized Difference Built-up Index \& Normalized Difference Vegetation Index (NDBI \& NDVI)}

The normalized difference built-up index (NDBI) is used to extract built-up features which ranges from -1 to 1 . The normalized difference vegetation index (NDVI) is used to monitor the condition of vegetation and is the most commonly used vegetation index globally. A study from Malik, Shukla \& Mishra (2018) shows that the positive relationship found between NDBI and LST indicates that the built-up areas are generating much surface temperature variations and is the key contributor in the UHI. On the other hand, healthy vegetative cover plays a key role in lowering LST.

\section{METHODOLOGY}

The methodology for the geospatial assessment and modelling of urban heat islands (UHI) in Quezon City is shown in Fig. 1.

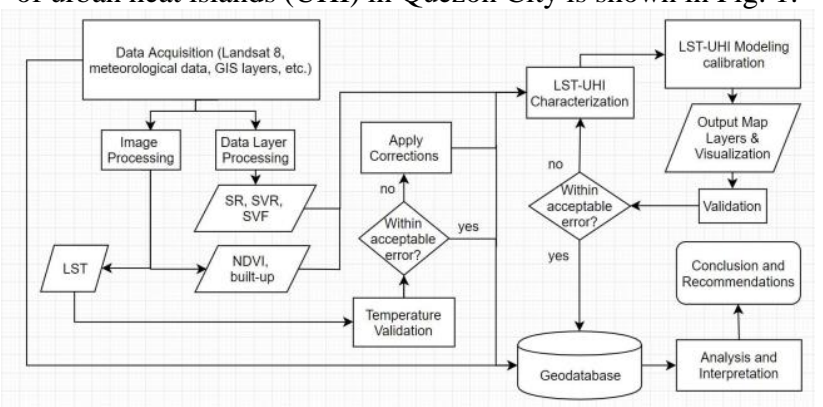

Figure 1. General Methodology Flowchart

Data preparation includes data acquisition and processing of satellite images. The Landsat 8 satellite images are processed using Google Earth Engine. The spatial and temporal resolution for the Landsat data for deriving LST data is $100 \mathrm{~m}$ and once every 16 days, respectively. The dates used for modelling is chosen by least cloud cover that dates February 5, 2019 and August 10, 2017. The months were chosen in order to compare the wet and dry seasons of the year.

The data are then examined in order to determine the UHIs in Quezon City. Clusters of high-temperature pixels would be one of the main bases for classifying an area as a UHI. Different characteristics (e.g., surface albedo, sky-view factor, surface area-to-volume ratio) are considered by determining their relationships with the intensity of the UHI in a given area. Regression analyses were conducted in order to model the UHI using different variables. Each of the six factors, namely, SR, SVR, SVF, SA, NDBI and NDVI, are used as explanatory variables to describe the variations of LST. 


\subsection{Image Pre-processing \& LST Calculation}

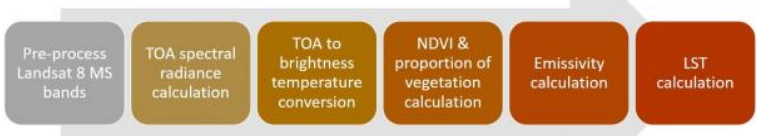

Figure 2. Image Pre-processing Workflow

LST based on Landsat 8 data can be calculated (Jeevalakshmi et al., 2018) using formula 1 :

$$
\mathrm{T}_{\mathrm{S}}=\frac{\mathrm{BT}}{\left\{1+\left[\left(\frac{\lambda \mathrm{BT}}{\rho}\right) \ln \varepsilon_{\lambda}\right]\right\}}
$$

Where:

$$
\begin{aligned}
& \mathrm{T}_{\mathrm{s}}=\mathrm{LST} \text { in degrees Celsius } \\
& \mathrm{BT}=\text { brightness temperature } \\
& \lambda=\text { avg. wavelength of band } 10= \\
& 10.89 \varepsilon_{\lambda}=\text { Land Surface Emissivity } \\
& \rho=[\mathrm{h} \times(\mathrm{c} / \sigma)]=1.438 \times 10^{-2} \mathrm{mK} \\
& \mathrm{h}=6.62607004 \times 10-34 \mathrm{~m}^{2} \mathrm{~kg} / \mathrm{s} \\
& (\mathrm{Planck} \mathrm{s} \text { constant) } \\
& \mathrm{c}=\text { speed of light } \sim 3.00 \times 108 \mathrm{~m} / \mathrm{s} \\
& \sigma=1.38064852 \times 10^{-23} \mathrm{~m}^{2} \mathrm{~kg} \mathrm{~s}^{-2} \mathrm{~K}^{-1} \\
& \text { (Boltzmann Constant) }
\end{aligned}
$$

\subsection{Surface Albedo (SA) Calculation}

Surface albedo based on Landsat 8 data can be calculated (Baldinelli et al., 2017) using equation 2

$$
S A=c_{0}+c_{1} B_{1}+c_{2} B_{2}+\cdots+c_{7} B_{7}
$$

Where: $\quad \mathrm{SA}=$ Surface Albedo

$\mathrm{B}_{\mathrm{i}}$ are band values.

\begin{tabular}{|l|l|l|l|l|l|l|l|l|}
\hline Coefficient & $\mathrm{C}_{0}$ & $\mathrm{C}_{1}$ & $\mathrm{C}_{2}$ & $\mathrm{C}_{3}$ & $\mathrm{C}_{4}$ & $\mathrm{C}_{5}$ & $\mathrm{C}_{6}$ & $\mathrm{C}_{7}$ \\
\hline Value & 0.043 & 0.082 & 0.064 & 0.173 & 0.114 & 0.237 & 0.252 & 0.034 \\
\hline
\end{tabular}

Table 1. Coefficients used for SA calculation

\subsection{Urban thermal field variance index (UTFVI)}

Urban thermal field variance index is a quantitative measure that defines how severe UHI is on an area. Equation 3 shows how to calculate UTFVI.

$$
\text { UTFVI }=\frac{\text { Ts-Tmean }}{\text { Ts }}
$$

Where:

$U T F V I=$ Urban thermal field variance index

$T s=\mathrm{LST}$ of a certain point in Kelvin

Tmean $=$ Mean LST of the subject area

The measures can be categorized as follows to determine the intensity and qualify the values derived according to the strength of UHI in the area, as determined by Zhang (2009), and is summarized in Table 2.

\begin{tabular}{|c|c|}
\hline $\begin{array}{c}\text { Urban Thermal Field } \\
\text { Variance Index }\end{array}$ & UHI Strength \\
\hline$<0$ & None \\
\hline $0.000-0.005$ & Weak \\
\hline $0.005-0.010$ & Moderate \\
\hline $0.010-0.015$ & Strong \\
\hline $0.015-0.020$ & Stronger \\
\hline 0.020 & Strongest \\
\hline
\end{tabular}

Table 2. UHI Strength based of UTFVI

\subsection{Feature data extraction from DSM, DTM and shapefiles}

Building geometry measurements were calculated in ArcGIS. This is necessary for estimating the factors in UHILST model such as the SVR, SVF and SR.

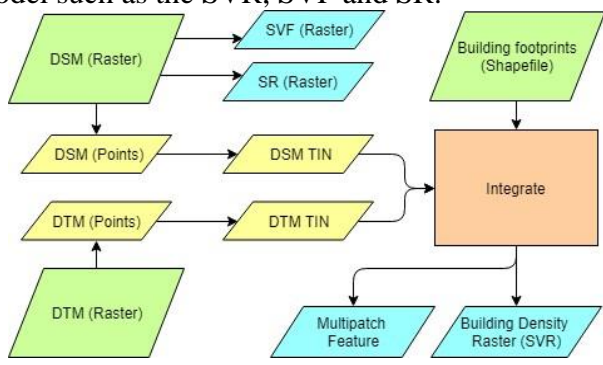

Figure 3. Feature data extraction workflow

The feature datasets are validated for accuracy and completeness. A common reference system is be used to project them, and satellite images from Google Earth are used as reference.

The sky view factor (SVF) was calculated using the built-in tool in the SAGA GIS open source software by using the DSM raster as input. The solar radiation (SR) also uses the DSM raster as input for the spatial analyst tool for solar radiation in ArcGIS. Cumulative SR was computed from 6:00 AM to 10:30 AM, which close to the Landsat 8 acquisition time.

Surface-Area-to-Volume Ratio (SVR) was derived from the multipatch features created by using the Extrude Between tool (3D analyst) of ArcGIS, for which the input features are two triangulated irregular networks (TINs) generated from DSM and DTM datasets. The multipatch features were converted back to 2D polygon feature and the SVR raster was created.

The data, including the raster data sets and the DTM and DSM files are integrated in ArcGIS. A fishnet is created with a pixel size of 30 by $30 \mathrm{~m}$. resolution to match the spatial resolution of the initial Landsat 8 data. Zonal statistics is performed for all the raster variables after they have the same resolution to generate a table of attributes which contains the height, LST, albedo and other factors related to UHI.

\subsection{Ordinary Least Squares (OLS) Regression}

OLS model describing LST variations using NDVI, NDBI, SR, SVR, SVF and surface albedo. The model is examined using $R_{2}$, Adjusted $\mathrm{R}_{2}$, and RMSE to evaluate the overall model performance. The significance of the Koenker (BP) statistic and the Jarque-Bera statistic are evaluated to determine nonstationarity of the model and the non-normality in the distribution of residuals, respectively.

\subsection{Geographically Weighted Regression (GWR)}

If the process is nonstationary as indicated by the significance of the Koenker (BP) statistics, GWR analysis is performed to create numerous local models of LST variations. GWR enables the coefficients of factors to vary in space to account for nonstationarity. Three models at different bandwidth distances were developed in order to determine the best parameters that would create the best fit. The parameters of each model are stated below:

Dependent Variable: LST 
The International Archives of the Photogrammetry, Remote Sensing and Spatial Information Sciences, Volume XLII-4/W16, 2019 6th International Conference on Geomatics and Geospatial Technology (GGT 2019), 1-3 October 2019, Kuala Lumpur, Malaysia

Explanatory Variables: SVF, SR, SVR, SA, NDBI, NDVI

Kernel type: Adaptive

Bandwidth Method: Bandwidth Parameter

Distance (m): 30, 100, and 200

\section{RESULTS AND DISCUSSION}

\subsection{Urban Thermal Characteristics and Patterns}

In order to characterize the thermal characteristics and patterns in Quezon City, analysis of LST in relation to land use was conducted. As can be seen in Figs 4 and 5, industrial, commercial, and residential areas are relatively hotter with average temperature of around $33.5^{\circ} \mathrm{C}$. This is in contrast to lower average surface temperatures of parks $\left(30.5{ }^{\circ} \mathrm{C}\right)$, agriculture and water areas $\left(26^{\circ} \mathrm{C}\right)$, and even institutional areas $\left(32^{\circ} \mathrm{C}\right)$. Within each land use types, LST can be seen to vary greatly.

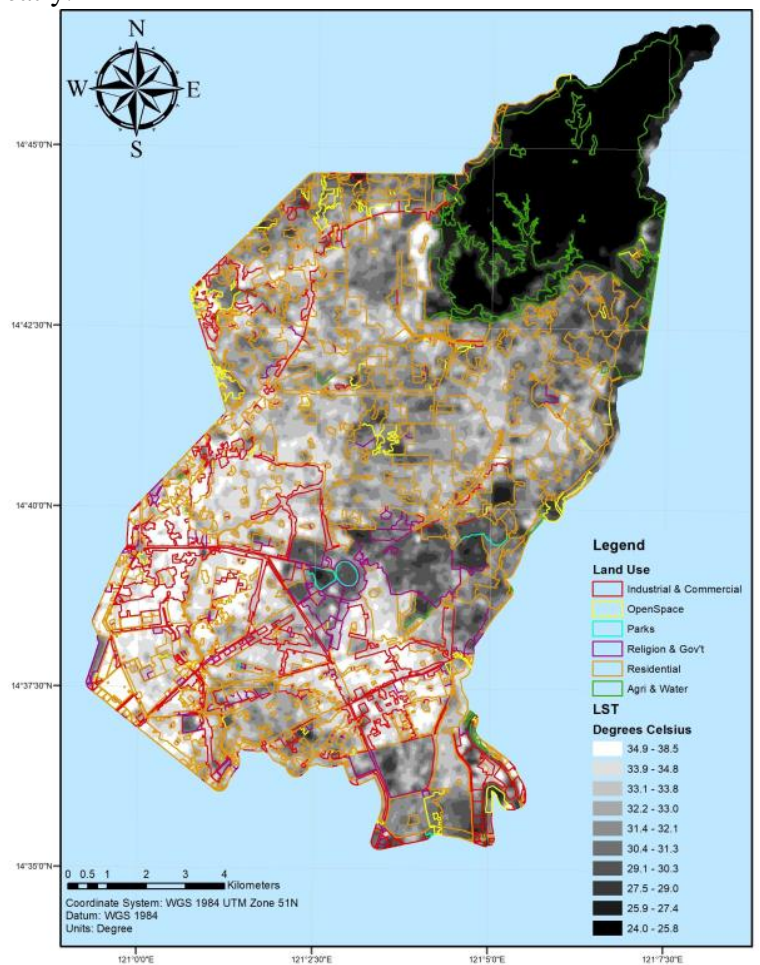

Figure 4. Land surface temperature (LST) distribution in Quezon City on 5 February 2019

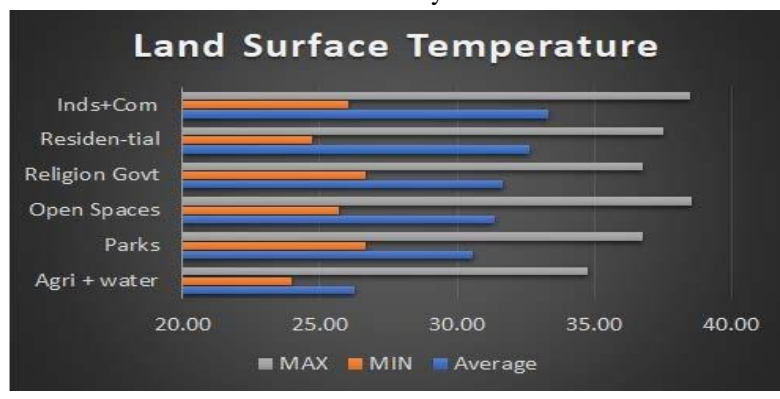

Figure 5. Minimum, maximum, and average land surface temperature per land use type in Quezon City on 5 February 2019

\subsection{UHI Analysis}

Urban Heat island has been categorized in terms of intensities using the Urban Thermal Field Variance Index (UFTVI) (Fig.
6). UFTVI as described by Table 2 shows the different strengths or intensities of UHI. Evidently, stronger UHIs are found in residential, commercial and industrial areas. These areas are typically dominated by impervious surfaces and have less vegetation compared to other areas. Variations of UHI intensity in these areas can also be attributed to the density and sizes of houses and buildings.

The barangays (villages) are classified and ranked based on UHI strength detected in the locality; the zonal mean of UTFVI were computed per barangay. In Fig. 7, the northeastern and central eastern barangays have the lowest UHI strength. Barangays with higher UHI strength can be found in the southwestern and southeastern parts. These observations are consistent with the LST distribution.

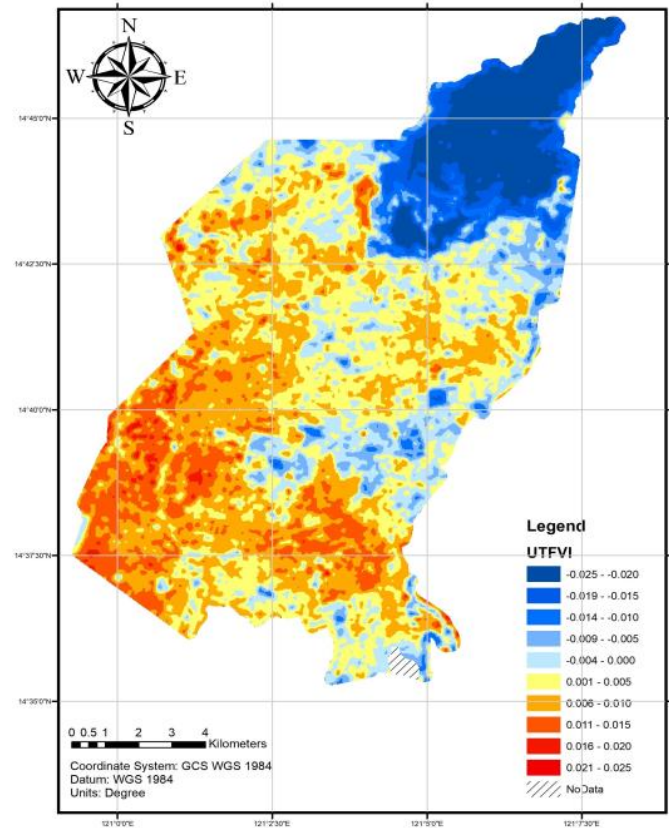

Figure 6. Spatial variation of UTFVI-based UHI intensity in Quezon City on 5 February 2019

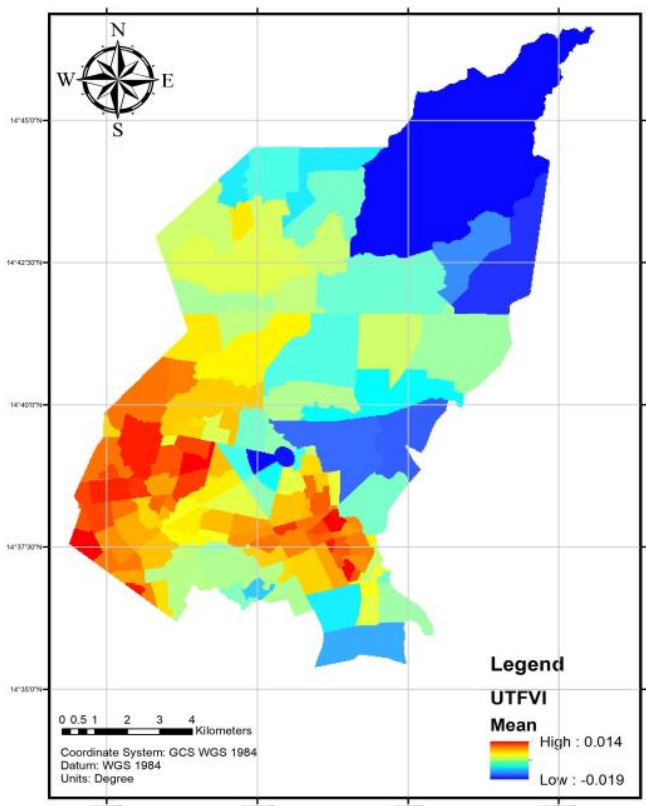

Figure 7. Mean UTFVI per barangay in Quezon City for 5 February 2019 
The top 10 identified barangays all lie on the southern half of Quezon City while the bottom 10 lie on the extreme northeastern, extreme southeastern and central northern parts. The normalized mean for the UTFVI, SR, SVF, SVR, SA, NDBI \& NDVI were computed for the whole of QC, top 10 \& bottom 10 barangays, with normalization based from the maximum values for each factor for the entire city (see Fig. 8). Each mean value is shown in percentage relative to the maximum of each variable for better visualization.

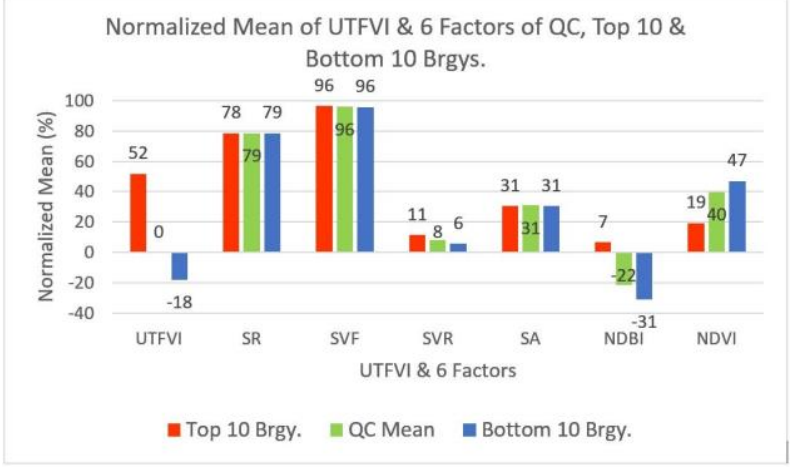

Figure 8. Normalized mean of UTFVI, SR, SVF, SVR, SA, NDBI \& NDVI for Quezon City, the top 10, and bottom 10 barangays based mean UTFVI in Quezon City for 5 February 2019

The mean UTFVI for the top 10 barangays (52\%) is much higher compared to the mean UTFVI of QC (0) and mean UTFVI of the bottom 10 (18). The SR \& SVF mean values for the three categories does not have a significant difference when normalized using the maximum value for Quezon City. The difference of 0.395 between the top \& bottom 10 SR values is insignificant compared to their mean magnitudes of above 259 . The difference of 0.006 for the SVF of the top \& bottom is very small compared to their average magnitude of 0.961 . The values for SVR decreases from top 10 to mean to bottom 10. The SVR for the top 10 barangays is higher than the average SVR of QC by $3 \%$, while the bottom 10 is lower than $2 \%$ from the average.

The SA for the bottom 10 and the top 10 barangays are the same, having a value of 0.002 lower than the average of the whole of QC. The NDBI, like the SVR, also decreases from top 10 to bottom 10, making a huge difference from the average of $29 \%$. The NDBI of the bottom 10 when compared to the average makes a difference of almost 9\% lower than the average. The difference between the top and the bottom 10 is almost 38\%, making the largest difference between all the factors excluding UTFVI. Unlike the trends for the SVR and NDBI which decreases, the NDVI increases from the top 10 to the bottom 10, showing an inverse trend when compared to the UTFVI or UHI strength. The difference between the NDVI values for the top 10 is $11 \%$ lower than the average while for the bottom 10 , it is $7 \%$ higher than the average. The difference between the top 10 and the bottom 10 is $28 \%$, making the 2 nd largest difference between all factors excluding the UTFVI next to the NDBI.

\subsection{OLS Regression Model for LST}

All the variables, namely, SR, SVF, SV, SA, NDVI and NDBI, have significant $(\mathrm{p}<.01)$ correlation with LST (Fig. 9). The variable with the most linear fit is the NDBI, with an $\mathrm{R}_{2}$ value of 0.65 , therefore explaining $65 \%$ of the variability of LST. NDVI has a negative slope, which explains $41 \%$ of the variability of LST. The other factors might not indicate strong linear relationship with LST, but they can be significant in modelling LST. All six factors were therefore used in order to model LST using OLS regression. Results are shown in Tables 3 and 4. There is no global multicollineary as indicate by the variance inflation factor (VIF) values, which are all less than 7.5. All variables are significant components of the model as indicated by the robust probability (Robust_Pr). It should be noted that the Koenker (BP) and the Jarque-Bera statistic are both significant. When the Koeker BP is significant, it means that the modeled relationships are not consistent either due to nonstationatity or heteroskedasticity. When Jarque-Bera is significant, model predictions are biased and the residuals are not normally distributed.

\section{Variable Coefficient StdError t-Statistic Probability} Robust_SE Robust_t Robust_Pr VIF

Table 3. Summary of ordinary least squares results - model variables for LST regression model in Quezon City for 5 February 2019

\begin{tabular}{r|r|r|r|l|l|r|r|r|}
\hline Intercept & 32.098 & 0.119 & 268.777 & $0.000 *^{*}$ & 0.123 & 260.094 & $0.0000^{*}$ & ----- \\
$S R$ & & & & & & & & - \\
$S V F$ & -0.001 & 0.000 & -6.707 & $0.0000^{*}$ & 0.000 & -6.674 & $0.0000^{*}$ & 1.430 \\
$S V R$ & 0.869 & 0.144 & 6.053 & $0.0000^{*}$ & 0.149 & 5.846 & $0.0000^{*}$ & 1.477 \\
& 1.066 & 0.009 & 114.741 & $0.0000^{*}$ & 0.014 & 75.150 & $0.0000^{*}$ & 1.348 \\
$S A$ & 1.092 & 0.152 & 7.170 & $0.0000^{*}$ & 0.210 & 5.187 & $0.0000^{*}$ & 1.211 \\
$N D B I$ & 11.969 & 0.039 & 310.199 & $0.0000^{*}$ & 0.109 & 110.144 & $0.0000^{*}$ & 2.410 \\
$N D V I$ & -2.299 & 0.024 & -96.203 & $0.0000^{*}$ & 0.055 & -41.927 & $0.0000^{*}$ & 2.101
\end{tabular}

\begin{tabular}{|c|c|c|c|}
\hline $\begin{array}{l}\text { Number of } \\
\text { Observations: }\end{array}$ & 194869 & Akaike's Information Criterion (AICc): & 754376.9 \\
\hline Multiple R-Squared: & 0.69097 & Adjusted R-Squared: & 0.69096 \\
\hline Joint F-Statistic: & 72616.35 & $\operatorname{Prob}(>\mathrm{F}),(6,194862)$ degrees of freedom: & 0.000000 \\
\hline Joint Wald Statistic: & 321523.1 & Prob(>chi-squared), (6) degrees of freedom: & $0.000000^{*}$ \\
\hline $\begin{array}{l}\text { Koenker (BP) } \\
\text { Statistic: }\end{array}$ & 6220.422 & Prob(>chi-squared), (6) degrees of freedom: & $0.000000^{*}$ \\
\hline $\begin{array}{l}\text { Jarque-Bera } \\
\text { Statistic: }\end{array}$ & 37296.66 & Prob(>chi-squared), (2) degrees of freedom: & 0.000000 \\
\hline
\end{tabular}
in Quezon City for 5 February 2019

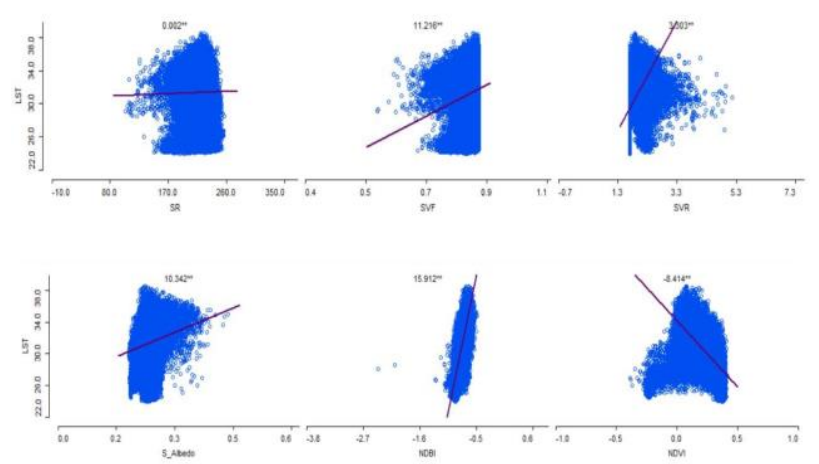

Figure 9. Scatter plot diagrams (left to right) for LST vs. SR, SVF, SVR, SA, NDBI, and NDVI. The lines show the linear relationship between the variables listed. 


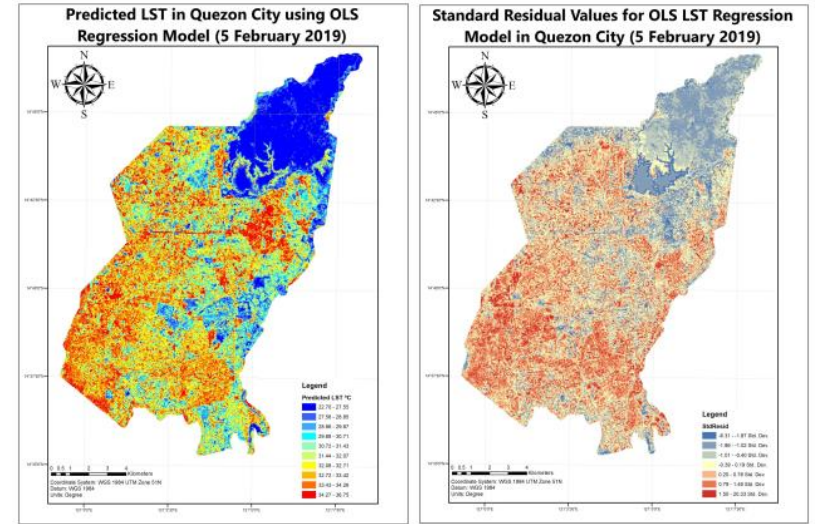

Figure 10. Ordinary least squares predicted values (left) and Standard Residuals (right) for LST regression model in Quezon City for 5 February 2019

The Root Mean Squared Error of the model is also computed with a value of 1.67. The standard deviations of the residuals are mapped to determine how much the predicted values differ from the 'Observed' values. According to the map, red areas show high positive values of error or overprediction and blue areas show negative values of error or underprediction. The residuals exhibit clustering in space. The OLS regression residuals are large and does not strictly follow a normal distribution (Fig.11). This is a violation of the assumption of normality in OLS. This means that the model does not fully represent or explain the whole data set. The solution is to find a model that would generate a residual plot that has a bell-shaped curve. The significance of the Koenker (BP) indicates the need to proceed to spatial regression (e.g., GWR) to address non-stationarity.

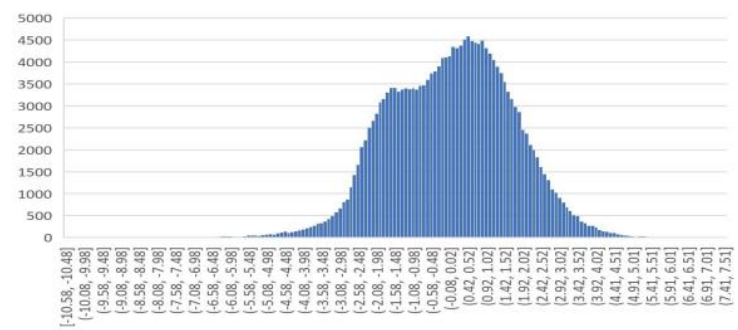

Figure 11. Histogram of OLS Regression Residuals

\subsection{Geographically Weighted Regression Model for LST}

The GWR model is an outgrowth of the OLS, which accounts for its variation by locality. Since the model is non-stationary, application of GWR is fit because it is a technique that accounts for non-stationary variables and takes them into consideration (Charlton \& Fotheringham, 2009). After checking the statistical results of the OLS regression model, and passing the tests, the next step is to proceed to the GWR model. Similar to the OLS model for LST, only the February 5 LST map was predicted by the model because of the aforementioned reasons. To acquire the best model for the LST using GWR, three maps were generated each having unique parameters in ArcMap.

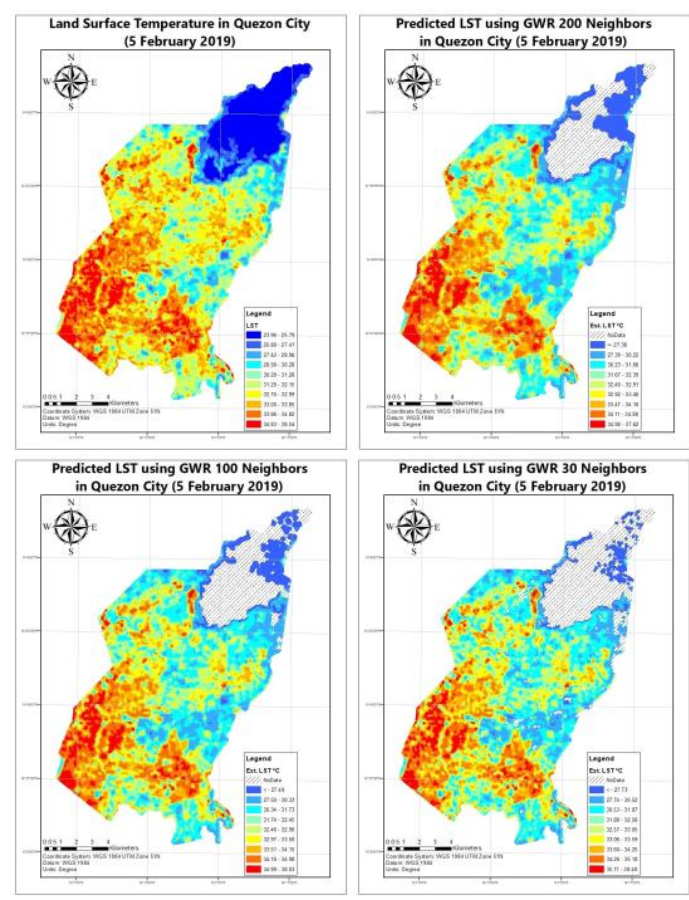

Figure 12. From left to right, LST map and Predicted LST using GWR (200); GWR (100); GWR (30).

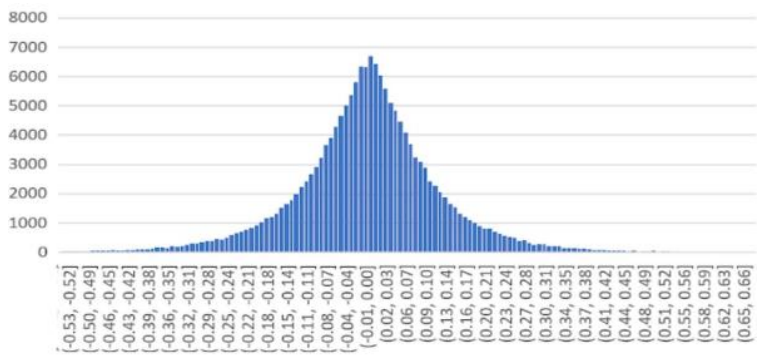

Figure 13. Histogram of GWR 30 Residuals

As compared with the residual plot of the OLS regression, the residuals of the GWR model displays a normal distribution (Fig. 13). The GWR models explains LST better judging from the low residuals.

To further understand the results, a report was generated in ArcMap, as shown in Table 4. Bandwidth Parameter dictates how many neighbors are going to be considered by the GWR model in its calculations. The values considered for the models are 30 (GWR_30), 100 (GWR_100), and 200 (GWR_200) as previously mentioned.

\begin{tabular}{|l|c|c|c|}
\hline & GWR 30 & GWR 100 & GWR 200 \\
\hline $\begin{array}{l}\text { Bandwidth } \\
\text { Parameter }\end{array}$ & $\begin{array}{c}\text { 30 } \\
\text { Neighbors }\end{array}$ & $\begin{array}{c}100 \\
\text { Neighbors }\end{array}$ & $\begin{array}{c}200 \\
\text { Neighbors }\end{array}$ \\
\hline Residual Squares & $\mathbf{0 . 4 5 2}$ & 9.519 & 18.144 \\
\hline Sigma & $\mathbf{0 . 2 9 5}$ & 0.547 & 1.632 \\
\hline AICc & 35.647 & 75.611 & $\mathbf{3 5 . 1 5}$ \\
\hline Ave. of Local R2 & $\mathbf{0 . 5 5 1 7}$ & 0.4183 & 0.3942 \\
\hline RMSE & $\mathbf{0 . 1 4}$ & 0.26 & 0.39 \\
\hline
\end{tabular}

Table 5. GWR Diagnostics for LST models in QC for Feb. 5, 2019 
Residual Squares is the sum of the squared residuals generated in the model. Better resulting model are observed when this value is closer to 0 . The GWR_30 has the least value for the residual squares with a value of 0.452 which is significantly better compared to the other 2 models. Sigma is the standard deviation of the residuals. A small deviation of the residuals signifies that the prediction is close to the observed values. GWR_30 gives the best value of sigma of 0.295, this means that 1 standard deviation within the model is equal to $0.295^{\circ} \mathrm{C}$ of difference from the observed LST.

AICc determines the relative performance of the model. The model with the significantly lower AICc value provides a better fit to the observed data. GWR_200 has the least AICc value however it is almost equivalent to the AICc of GWR_30. In terms of AICc, GWR_30 and GWR_200 are equal in model performance. Upon trials of the neighbors beyond 200, the AICc's of the models tend to grow bigger continuously. The Local $\mathrm{R}_{2}$ measures the goodness of the fit with a range of values from 0.0 to 1.0. If the value is closer to 1 , the model represents the real values more accurately. The measure is local because it only computes its fit depending on the number of neighbors it encloses. GWR_30 produced the highest average Local $R^{2}$ at 0.5517. The RMSE is the square root of the average of the squares of all residuals in each model and gives more weight to larger residuals. GWR_30 yields the lowest RMSE with 0.14.

The variation of GWR variable coefficients vis-à-vis index values is worth looking into. Figure 14 shows a sample of NDBI coefficients, NDBI values and corresponding true color image.

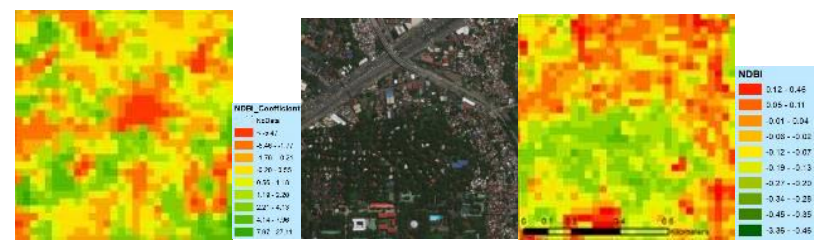

Figure 14. Sample GWR NDBI coefficients, true color image and NDBI values

The negative values of NDBI Coefficient are typically seen in the regions where there are fewer buildings. Positive values of NDBI Coefficients are seen on the areas where there are high number or density of buildings and houses thus yielding a more positive value for the equation, therefore increasing the total value of LST for that specific area.

NDVI on the other hand, decreases the total LST of a point or an area depending on how much trees or vegetation therein. This implies that the total LST will decrease by the value that was obtained by crossing the negative NDVI coefficient and the high positive NDVI value for areas that are rich in vegetation.

\subsection{LST Calculations}

From the same region as above, one representative pixel value for each highly vegetated area and not vegetated area is selected for the sample LST calculations.
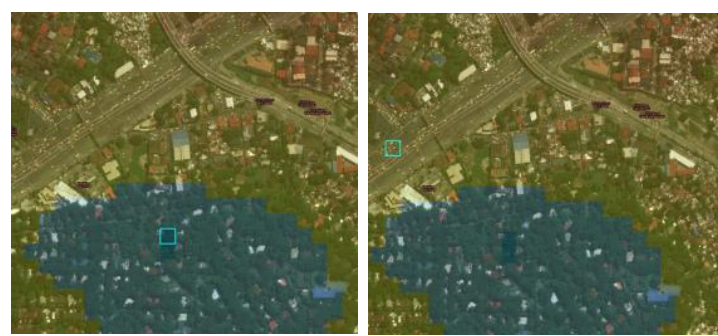

Figure 15. Sample stacked true color image and LST image of tree pixel (left, FID = 72220) and road pixel (right, FID = 73559)

\begin{tabular}{l|l|l|l|l|l|l|} 
FID & SR & SVF & SVR & S_Albedo NDBI & NDVI & LST
\end{tabular}

\begin{tabular}{|l|r|r|r|r|r|r|r|}
\hline 72220 & 267.56 & 0.9745 & 0.501 & 0.15868 & -0.28971 & 0.63108 & 28.3428 \\
\hline
\end{tabular}

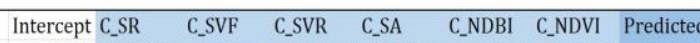
$\begin{array}{lllllllll}\text { GWR } 30 & 32.0626 & 0.00312 & -3.78398 & -0.27567 & -0.15024 & -0.79706 & -1.31682 & 28.449\end{array}$ $\begin{array}{llllllllll}\text { GWR } 100 & 32.8474 & -0.00363 & 0.26967 & -0.29743 & -8.10149 & 0.42106 & -3.04149 & 28.6619\end{array}$ \begin{tabular}{lllllllll} 
GWR 200 & 34.9498 & -0.00408 & 0.81667 & 0.06768 & -17.2928 & 1.42937 & -4.21236 & 28.8717 \\
\hline
\end{tabular}

Table 6. Pixel values and its computed coefficients for LST GWR models for tree pixel

\begin{tabular}{|l|l|l|l|l|l|l|}
\hline \multicolumn{1}{|c|}{ 72220 } & SR & SVF & \multicolumn{1}{l|}{ SVR } & \multicolumn{1}{l|}{ S_Albedo } & NDBI & NDVI \\
\hline GWR 30 & 0.83586 & -3.68748 & -0.13811 & -0.02384 & 0.23092 & -0.83102 \\
GWR 100 & -0.97231 & 0.26279 & -0.14901 & -1.28554 & -0.12198 & -1.91942 \\
GWR 200 & -1.09138 & 0.79584 & 0.03391 & -2.74402 & -0.4141 & -2.65833 \\
\hline \hline
\end{tabular}

Table 7. Computed contribution of variables to LST in ${ }^{\circ} \mathrm{C}$ of tree pixel (values in ${ }^{\circ} \mathrm{C}$ )

\begin{tabular}{|c|c|c|c|c|c|c|c|c|}
\hline \multirow{2}{*}{\multicolumn{2}{|c|}{$\begin{array}{c}\text { FID } \\
73599\end{array}$}} & \multirow{2}{*}{\begin{tabular}{|l|} 
SR \\
275.313 \\
\end{tabular}} & \multirow{2}{*}{\begin{tabular}{|l|} 
SVF \\
0.90686
\end{tabular}} & \multirow{2}{*}{ 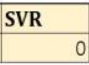 } & \multicolumn{2}{|c|}{ S_Albedo NDBI } & \multirow{2}{*}{\begin{tabular}{|l|l} 
NDVI \\
$5.010 \mathrm{~s}$
\end{tabular}} & \multirow{2}{*}{\begin{tabular}{|l} 
LST \\
32.1945
\end{tabular}} \\
\hline & & & & & \begin{tabular}{l|l|} 
& 0.1437
\end{tabular} & 0.02735 & & \\
\hline & Intercept & C_SR & C_SVF & C_SVR & C_SA & C_NDBI & C_NDVI & Predictec \\
\hline GWR 30 & 31.4259 & 0.00036 & 0.02599 & 0.58996 & 1.78795 & 5.35138 & 0.31593 & 31.9559 \\
\hline GWR 100 & 30.5609 & 0.00577 & -0.57535 & 0.5383 & 2.49236 & 2.70237 & -1.24453 & 32.0459 \\
\hline GWR 200 & 29.1693 & 0.00595 & 0.95224 & 0.42523 & 2.74887 & 1.8761 & $1-2.19951$ & 32.0928 \\
\hline
\end{tabular}

Table 8. Pixel values and its computed coefficients for LST GWR models for road pixel

\begin{tabular}{|c|c|c|c|c|c|c|c|}
\hline 73599 & SR & SVF & SVR & \multicolumn{3}{|c|}{ S_Albedo NDBI } & NDVI \\
\hline GWR 30 & 0.09966 & 0.02357 & & 0 & 0.25693 & 0.14636 & 0.0038 \\
\hline GWR 100 & 1.58828 & -0.52176 & & 0 & 15 & 0.0 & -0.01357 \\
\hline GWR 200 & 1.63756 & 0.86355 & & 0 & 0.39501 & 0.05131 & -0.02397 \\
\hline
\end{tabular}

Table 9. Computed contribution of variables to LST in ${ }^{\circ} \mathrm{C}$ of road pixel (values in ${ }^{\circ} \mathrm{C}$ )

The values of the GWR coefficients of the variables were multiplied to the values of the variables in order to determine the actual contribution of each variable to the LST in ${ }^{\circ} \mathrm{C}$. Each model has a different intensity of coefficient for each variable. For the highly vegetated area, (72220 pixel), SVF and NDVI has the most contribution to the LST $\left(-3.8\right.$ and $-1.3{ }^{\circ} \mathrm{C}$ respectively) for GWR30. Note that these values are negative meaning, that the LST will be lower by increasing the NDVI and SVF.

Surface Albedo on the other hand, has the contributed most (-1.3 and $-2.7^{\circ} \mathrm{C}$ ) for the GWR 100 and GWR 200. For the road pixel, the most significant factor, using the GWR30 model, is the SA and NDBI with values of 0.25 and $.15^{\circ} \mathrm{C}$ respectively. Using GWR100 and GWR200, highest values are found in the SR with values 1.58 and $1.63{ }^{\circ} \mathrm{C}$ respectively. The SVR has a 0 value because of the absence of a building in the actual pixel. NDVI also has almost no significance to the total LST in the said pixel. 


\section{CONCLUSIONS AND RECOMMENDATIONS}

A methodology was developed and implemented to compute LST and model the UHI in Quezon City. The LST, NDVI, NDBI and SA bands were derived from Landsat 8 images. UTFVI was used to classify the strength of UHI in an area based on the mean temperature of the whole city. The factors considered in analysis are SR, SVF, SVR, SA, NDBI, and NDVI. The top 10 barangays according to UHI strength and the bottom 10 were analyzed. Significant differences between the top 10 and bottom 10 include a $38 \%$ discrepancy in their mean normalized NDBI, $-28 \%$ in their mean normalized NDBI, and $5 \%$ in their mean normalized SVR. The differences for the standardized mean of the remaining factors were insignificant.

The northeastern areas of Q.C. contain lower LST than the southwestern parts. Similar patterns for low to high values for NDBI were observed, while for the NDVI, the values appear in reverse, from high to low. Industrial and commercial land uses have the highest mean LST among all land use classes, partnered with a low minimum SVF and SR, high mean SVR, high mean NDBI and low mean NDVI and high max SA; while the agricultural and water classes have lowest mean LST partnered with an average minimum SR, high minimum SVF, low mean SVR, low mean NDBI, high mean NDVI and low maximum SA. The relationships established that are directly proportional to LST are SVR and NDBI, while the relationship for NDVI is inversely proportional, which is consistent with other studies.

Regression models were made for LST, considering all the six factors as explanatory variables. The first model was made using ordinary least squares technique. The explanatory variables explain $69 \%$ of the variability of LST based on the R2 value. Tests show that the factors are all significant and are not redundant. The scatter plot shows that NDBI has the best linear fit with the LST compared to all other factors. The spatial autocorrelation report for the residuals of this model shows a significant clustering, meaning that the residuals are not random, thus, to account for spatial autocorrelation and nonstationarity of values, a second model was produced using the geographically weighted regression technique. Three models with 30, 100 and 200 bandwidth parameters were produced using an adaptive kernel. The general pattern and appearance of the observed values for LST was captured by all three models. The deviations are more random using GWR. The best model which fits the observed LST is the one with 30 as bandwidth parameter.

\section{ACKNOWLEDGEMENT}

This research is supported by the DOST Philippine Council for Industry, Energy and Emerging Technology Research and Development (PCIEERD) under the Geospatial Assessment and Modelling of Urban Heat Islands in Philippine Cities (GUHeat) Project.

\section{REFERENCES}

American Meteorological Society. (n.d.). Surface Albedo.

Baldinelli, G., Bonafoni, S., Rotili, A., \& Verducci, P. (2017). Albedo and surface temperature relation in urban areas: Analysis with different sensors. 2017 Joint Urban Remote Sensing Event (JURSE). doi:10.1109/jurse.2017.7924612
CLEAR. (n.d.). Retrieved from https://www.newlearn.info/packages/clear/interactive/matrix/lev el_11_surface_are a_to_volume_ratio.html Fotheringham, A. S., Brunsdon, C., \& Charlton, M. (2002, October 11). Geographically Weighted Regression: The Analysis of Spatially Varying Relationships.

Hämmerle, M., Gál, T., Unger, J., \& Matzarakis, A. (2011). Comparison of models calculating the sky view factor used for urban climate investigations. Theoretical and Applied Climatology, 105(3-4), 521-527. doi:10.1007/s00704-011-04023

Malakar, N. K., Hulley, G. C., Hook, S. J., Laraby, K., Cook, M., \& Schott, J. R. (2018). An Operational Land Surface Temperature Product for Landsat Thermal Data: Methodology and Validation. IEEE Transactions on Geoscience and Remote Sensing, 56(10), 5717-5735. doi:10.1109/tgrs.2018.2824828

Malik, Subzar, M., Shukla, Prakash, J., Mishra, \& Satanand. (2019, January 01). Relationship of LST, NDBI and NDVI using Landsat-8 data in Kandaihimmat Watershed, Hoshangabad, India.

Mirzaei, P. A. (2015). Recent challenges in modeling of urban heat island. Sustainable Cities and Society,19, 200-206. doi:10.1016/j.scs.2015.04.001

Mishra, V., Ganguly, A. R., Nijssen, B., \& Lettenmaier, D. P. (2015). Changes in observed climate extremes in global urban areas. Environmental Research Letters,10(2), 024005. doi:10.1088/1748-9326/10/2/024005

Myronidis, D., \& Ioannou, K. (2018). Forecasting the Urban Expansion Effects on the Design Storm Hydrograph and Sediment Yield using Artificial Neural Networks. Water, 11(1), 31. doi:10.3390/w11010031

Oke, T. R. (1988). "Street Design and Urban Canopy Layer Climate." Energy Buildings 11: 103113. doi:10.1016/03787788(88)90026-6.

Reducing urban heat islands: Compendium of strategies. (2008). Washington, DC: Climate Protection Partnership Division, U.S. Environmental Protection Agency.

S, Trishita. (2017, August 09). Term Paper on Urban Heat Island (UHI) | Geography.

Schneider, A., \& Breitner, S. (n.d.). Temperature effects on health - current findings and future implications

Wang, Y., \& Akbari, H. (2014). Effect of Sky View Factor on Outdoor Temperature and Comfort in Montreal. Environmental $\begin{array}{lll}\text { Engineering } & \text { Science, } & 31(6),\end{array}$ doi:10.1089/ees.2013.0430

Villanueva, M. J. (2015). RS and GIS-based Assessment and Modeling of Urban Environmental Conditions for Sustainable Urban Landscape Development (Unpublished master's thesis). University of the Philippines-Diliman.

Zhou, B., Rybski, D., \& Kropp, J. P. (2017, July 06). The role of city size and urban form in the surface urban heat island.

Revised August 2019 